\title{
Asymptomatic infection after BNT162b2 mRNA COVID-19 vaccination in multiple sclerosis patient
}

\author{
Dejan Jakimovski $^{1,2} \cdot$ Bianca Weinstock-Guttman $^{1}$ (])
}

Received: 6 June 2021 / Accepted: 6 September 2021 / Published online: 16 September 2021

(c) Belgian Neurological Society 2021

\section{To the Editor:}

Since the start of coronavirus disease (COVID-2019) pandemic caused by the severe acute respiratory syndrome coronavirus-2 (SARS-CoV-2), there has been more than 160 million infection cases and more than 3.3 million deaths worldwide [1]. As a response to the pandemic, swift efforts towards discovery, production, and distribution of SARSCoV-2 vaccines have been undertaken. This has resulted with approval and implementation of half a dozen vaccines including mRNA-based (BNT162b2, mRNA-1273), viral vector-based (Ad26.COV2.S, ChAdOx1 nCov-19 and Gam-COVID-Vac), and inactivated-virus vaccines (BBIBPCorV). In particular, such vaccines are able to significantly lower the incidence of symptomatic and asymptomatic SARS-CoV-2 infections [2]. Data regarding the infectiousness of already-vaccinated people and the effectiveness of the vaccines in immunocompromised or vulnerable patients are still emerging.

Hereafter, we describe a case of asymptomatic SARSCoV-2 infection in fully vaccinated multiple sclerosis (MS) patient, her associated serostatus and epidemiological data regarding her infectiousness. A 51-year-old Caucasian female MS patient treated with natalizumab for the past 10 years (extended interval dosing, q8 weeks) was seen by the immediate care facilities due to open wound from a human bite. The injury occurred at work as a social worker taking care of young students. She was started on

Bianca Weinstock-Guttman

bw8@buffalo.edu

1 Jacobs Comprehensive MS Treatment and Research Center, Department of Neurology, Jacobs School of Medicine and Biomedical Sciences University at Buffalo, 1010 Main Street, Buffalo, NY 14202, USA

2 Buffalo Neuroimaging Analysis Center, Department of Neurology, Jacobs School of Medicine and Biomedical Sciences, University at Buffalo, State University of New York, Buffalo, NY 14203, USA oral antibiotics and screened for infectious agents, including COVID-19 antibody test which confirmed acute and first exposure to SARS-CoV-2 with increased levels of IgM antibodies and negative IgG antibodies. (Luminescencebased VITROS anti-SARS-CoV-2 IgG test, VITROS ECi/ $\mathrm{ECiQ} / 3600$ Immunodiagnostic Systems) Additional nasal swab for polymerase chain reaction (PCR) test which was positive for SARS-CoV-2 RNA confirming current and active infection. This event occurred after 1 month since administration of the second dose of BNT162b2 mRNA vaccine. Both vaccination sessions were uneventful with minor local injection-site reactions (arm soreness). The last natalizumab infusion was administered 1 week after the first dose of the BNT162b2 mRNA vaccine. Throughout the entire time and after the positive tests, the patient felt good with no reports of COVID-19-related symptoms.

Additional epidemiological survey and SARS-CoV-2 testing of close contacts was performed. No positive tests were noted among the patient's co-workers and close family members. Additional repeat serologic and nasopharyngeal testing was performed 2 weeks after the asymptomatic infection. The latest results demonstrated loss of the IgM antibodies and high titer of anti-spike $\operatorname{IgG}$ antibodies, indicating acquired immunity. The follow-up PCR test was negative for SARS-CoV-2 RNA.

Albeit anecdotal, this case report may provide a glimpse to the real-world effectiveness of SARS-CoV-2 vaccination, the measurement and clinical response of MS patients towards the vaccines, and the patient's infectiousness after full vaccination. First, our case is corroborating the vaccine utility to prevent severe COVID-19 by reducing or fully blocking COVID-19 symptomatology. Despite the lack of detectable anti-SARS-CoV-2 antibodies, the patient had highly favorable clinical outcome and low infectiousness. Although natalizumab is not associated with systemic immunosuppression, it has been previously associated with lower vaccine response [3]. In addition to the favorable demographic factors such as young age and female sex, 
MS patients treated with natalizumab commonly have good COVID-19 outcomes $[4,5]$. The protective effects can be mainly attributed to $\mathrm{T}$ cell-based vaccine response $\left(\mathrm{CD}^{+}\right.$ $\mathrm{T}$ cells primed towards SARS-CoV-2 infected cells) which effectively limits the viral replication and control further spread of the virus. Therefore, evidence of anti-antibodies is only one part of immune response towards the vaccine and future decisions should not be solely based on this test. Along those lines the Centers for Disease Control and Prevention (CDC) and other medical governing bodies do not recommend testing for humoral response or following-up mild COVID-19 infections in people who underwent full vaccination [6].

The findings indirectly provide encouraging data regarding the overall expectations of COVID-19 vaccination in MS patients and in particular in patients that use diseasemodifying therapies (DMT) that cause B-cell depletion or immune cell sequestration [7]. While recent studies have demonstrated low SARS-CoV-2 seroconversion after vaccination, these findings were uncommon in patients with natalizumab [8]. For example, data from 35 Italian MS centers has shown that natalizumab does not affect the antiSARS-CoV-2 antibody levels measured 4 weeks after the full vaccination course [9]. Similar results were seen in UK where $92 \%$ of natalizumab-treated MS patients had positive serostatus after the full vaccination course (56 seropositive and 5 seronegative patients) [10]. In contrast, our patient did not develop detectable IgG antibodies after her vaccination course. However, her good disease control can be attributed to sufficient and specific anti-SARS-CoV-2 immunity through T-cell activation which can be measured on IFN- $\gamma$ enzyme-linked immunosorbent assay and may provide additional protective immunity [11]. Moreover, the patient could already have pre-existing T-cell immunity towards other non-spike proteins that is based on the cross-reactivity between the SARS-CoV-2 and the common cold coronaviruses such as (HCoV)-OC43, HCoV-229E, HCoV-NL63 and HCoV-HKU1 $[12,13]$. A limitation to this case report is the lack of information regarding the specific SARS-CoV-2 variant which may circumvent the vaccine immunity. In conclusion, our case report indirectly corroborates the vaccine effectiveness despite the seronegative status. MS care providers should continue recommending SARS-CoV-2 vaccination regardless on which DMT is used. The aforementioned CDC guidelines regarding the vaccine efficacy in the general population may be directly applicable to the MS population.

\section{Declarations}

Conflict of interest Dejan Jakimovski has nothing to disclosure. Bianca Weinstock-Guttman has participated in speaker's bureaus and/or served as a consultant for Biogen, Novartis, Genzyme; Sanofi, Genentech, AbbVie, Bayer AG, and Celgene/BMS. Dr. Weinstock-Guttman also has received grant/research support from the agencies listed in the previous sentence as well as Mallinckrodt Pharmaceuticals, Inc. She serves in the editorial board for BMJ Neurology, Journal of International MS and CNS Drugs.

\section{References}

1. Dong E, Du H, Gardner L (2020) An interactive web-based dashboard to track COVID-19 in real time. Lancet Infect Dis 20(5):533-534

2. Angel $\mathrm{Y}$ et al (2021) Association between vaccination with BNT162b2 and incidence of symptomatic and asymptomatic SARSCoV-2 infections among health care workers. JAMA. https://doi.org/ 10.1001/jama.2021.7152

3. Jakimovski D et al (2020) Infections, vaccines and autoimmunity: a multiple sclerosis perspective. Vaccines (Basel) 8(1):50

4. Salter A et al (2021) Outcomes and risk factors associated with SARS-CoV-2 infection in a North American registry of patients with multiple sclerosis. JAMA Neurol 78(6):699-708

5. Sormani MP et al (2021) DMTs and Covid-19 severity in MS: a pooled analysis from Italy and France. Ann Clin Transl Neurol $8(8): 1738-1744$

6. Centers for Disease Control and Prevention (2021) Interim clinical considerations for use of COVID-19 vaccines. https://www.cdc. gov/vaccines/covid-19/info-by-product/clinical-considerations.html. Accessed 11 May 2021

7. Centonze D et al (2021) Disease-modifying therapies and SARSCoV-2 vaccination in multiple sclerosis: an expert consensus. J Neurol. https://doi.org/10.1007/s00415-021-10545-2

8. Achiron A et al (2021) Humoral immune response to COVID-19 mRNA vaccine in patients with multiple sclerosis treated with high-efficacy disease-modifying therapies. Ther Adv Neurol Disord 14:17562864211012836

9. Tallantyre EC et al (2021) COVID-19 vaccine response in people with multiple sclerosis. medRxiv. https://doi.org/10.1101/2021.07. 31.21261326

10. Sormani MP, Inglese M, Schiavetti I, Carmisciano L, Laroni A, Lapucci C, Da Rin G, Serrati C, Gandoglia I, Tassinari T, Perego G, Brichetto G, Gazzola P, Mannironi A, Stromillo ML, Cordioli C, Landi D, Clerico M, Signoriello E, Frau J, Ferrò MT, Di Sapio A, Pasquali L, Ulivelli M, Marinelli F, Callari G, Iodice R, Liberatore G, Caleri F, Repice AM, Cordera S, Battaglia MA, Salvetti M, Franciotta D, Uccelli A, Group, CovaXiMS Study (2021) Effect of SARS-CoV-2 mRNA vaccination in ms patients treated with disease modifying therapies. SSRN. https://doi.org/10.2139/ssrn.3886420

11. Kister I, Krogsgaard M, Mulligan MJ et al (2021) Preliminary results of ongoing, prospective study of antibody and T-cell responses to SARS-CoV-2 in patients with MS on ocrelizumab or other diseasemodifying therapies. Neurology 96(22):014. https://doi.org/10.1212/ WNL.00000000000012044

12. Mateus J et al (2020) Selective and cross-reactive SARS-CoV-2 T cell epitopes in unexposed humans. Science 370(6512):89-94

13. Klompus $\mathrm{S}$ et al (2021) Cross-reactive antibodies against human coronaviruses and the animal coronavirome suggest diagnostics for future zoonotic spillovers. Sci Immunol. https://doi.org/10.1126/ sciimmunol.abe 9950

Publisher's Note Springer Nature remains neutral with regard to jurisdictional claims in published maps and institutional affiliations. 